\title{
Sex specific differences in postprandial glycaemic responses to white rice-based meals combined with chicken, fat and vegetable
}

\author{
M. Sayegh ${ }^{1}$, L. Sun ${ }^{2}$, M.K. Leow ${ }^{2}$, C.J. Henry ${ }^{2}$, V. Ranawana ${ }^{1}$ and J.E. Drew ${ }^{1}$ \\ ${ }^{1}$ The Rowett Institute, University of Aberdeen, Foresterhill, Aberdeen, UK, AB25 2ZD, UK and \\ ${ }^{2}$ Clinical Nutrition Research Centre, Singapore Institute for Clinical Sciences, Brenner Centre for Molecular \\ Medicine, Singapore 117609.
}

Consumption of high glycaemic index (GI) carbohydrate foods can result in postprandial hyperglycaemia and an increased risk of cardiovascular disease (CVD) ${ }^{(1)}$. Previous studies report that co-ingestion of high GI foods with protein, fat or vegetables, either singly or in combination, reduce postprandial glycaemic responses $(\mathrm{GR})^{(2)}$ and CVD risk $^{(3)}$. Inter-individual variations in GR precludes conclusive evidence ${ }^{(2,3)}$.

Individual variation in GR was further investigated using data from an acute randomised controlled crossover study, the Singapore study $^{(2)}$. Secondary data analysis was conducted on Singapore Study participants' $(n=14)$ postprandial GR at $0,15,30,45,60,90$, 120, 150 and 180 minutes following consumption of glucose or white rice-based meals combined with chicken, fat or vegetable. Principal component analysis (PCA) (Umetrics SIMCA-P + 12.0 software) was performed on the GR data. Further comparisons of the study data was conducted using ANOVA and independent samples t-test (ver. 24.0; SPSS Inc., Chicago, IL, USA).

A PCA plot (PC1 and PC2) accounting for $38 \%$ of the variation identified sex as a factor contributing to postprandial GR. Repeated measures ANOVA of postprandial GR at 0, 15, 30, 45, 60, 90, 120, 150 and 180 minutes confirmed significant differences between females $(n=6)$ and males $(n=8)$ following white rice consumption in combination with vegetable $(P=0.02)($ Fig 1 A) or chicken $(P=0.026)$ (Fig 1B). Independent sample t-tests, revealed statistically significant differences in GR between males and females at different time points (Figure 1). A trend $(\mathrm{P}=0.058)$ in $\mathrm{GR}$ differences between female and male cohorts consuming white rice co ingested with chicken, fat and vegetable was significant following post hoc tests at $60(\mathrm{P}=0.013)$ and 90 min $(\mathrm{P}=0.005)$. Females exhibited greater duration of elevated postprandial plasma glucose compared to males (Figure 1).
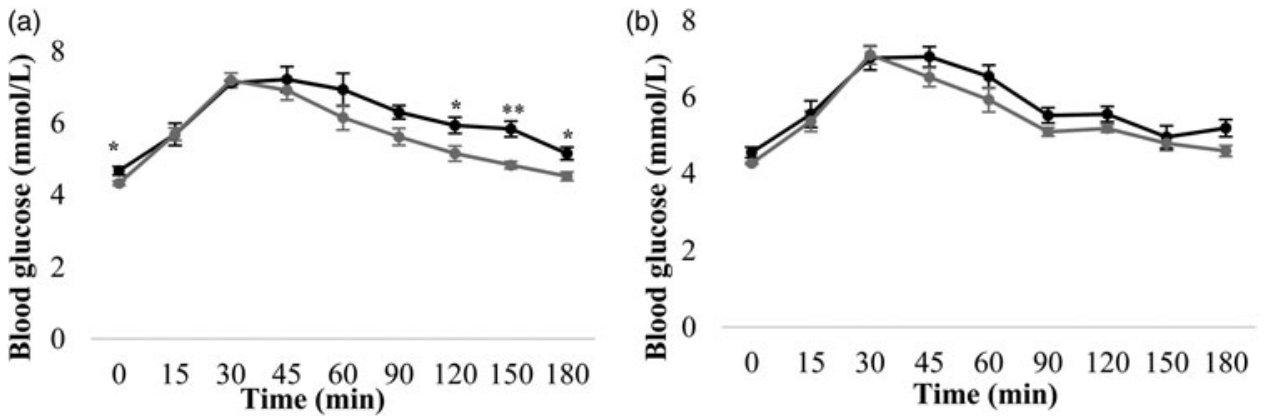

Fig. 1A-B. Mean postprandial GR in females (black) and males (grey) following consumption of white rice combined with vegetables (A) and white rice combined with chicken $(\mathrm{B})$ at $0,15,30,45,60,90,120,150$ and 180 min. Horizontal lines represent $\pm \mathrm{SE}$. ${ }^{*} \mathrm{P} \leq 0.05 ; * * \mathrm{P} \leq 0.001$ using independent samples t-test.

Further studies focusing on sex specific and inter-individual variation in GRs to meal combinations are required to identify links with CVD and effective dietary interventions to alleviate postprandial hyperglycaemia.

This work was supported by Scottish Government's Rural and Environment Science and Analytical Services Division (RESAS).

1. Woerle HJ, Neumann C, Zschau S, et al. (2007) Diabetes Res Clin Pract 77, 280-285.

2. Sun L, Ranawana DV, Leow MK, Henry CJ (2014) Eur J Nutr 53(8):1719-1726.

3. Imai S \& Kajiyama S (2010) Rehabil Health Sci 8, 1-7. 